\title{
Full-Thickness Resection Device for Complex Colorectal Lesions in High-Risk Patients as a Last-Resort Endoscopic Treatment: Initial Clinical Experience and Review of the Current Literature
}

\author{
Edris Wedi', Beatrice Orlandini ${ }^{2}$, Mark Gromski ${ }^{3}$, Carlo Felix Maria Jung ${ }^{1}$, rina Tchoumak', Stephanie Boucher ${ }^{4}$, Volker Ellenrieder ${ }^{1}$ and \\ Jürgen Hochberger ${ }^{5}$ \\ ${ }^{1}$ Department of Gastroenterology and Gastrointestinal Oncology, University Medical Center Göttingen, Göttingen, Germany, ${ }^{2}$ Department \\ of Gastroenterology, Careggi University Hospital, Florence, Italy, ${ }^{3}$ Division of Gastroenterology, Indiana University School of Medicine, \\ Indianapolis, IN, USA, ${ }^{4}$ Department of Pathology, Nouvel Hôpital Civil, Strasbourg University Hospitals, Strasbourg, France, ${ }^{5}$ Department of \\ Internal Medicine, Vivantes Klinikum im Friedrichshain, University Teaching Hospital of Humboldt University Berlin (Charité), Berlin, Germany
}

The full-thickness resection device (FTRD) is a novel endoscopic device approved for the resection of colorectal lesions. This case-series describes the device and its use in high-risk patients with colorectal lesions and provides an overview of the potential indications in recently published data.

Between December 2014 and September 2015, 3 patients underwent endoscopic full thickness resection using the FTRD for colorectal lesions: 1 case for a T1 adenocarcinoma in the region of a surgical anastomosis after recto-sigmoidectomy, 1 case for a non-lifting colonic adenoma with low-grade dysplasia in an 89-year old patient and 1 for a recurrent adenoma with high-grade dysplasia in a young patient with ulcerative rectocolitis who was under immunosuppression after renal transplantation. Both technical and clinical success rates were achieved in all cases. The size of removed lesions ranged from 9 to $30 \mathrm{~mm}$.

Overall, the most frequent indication in the literature has been for lifting or non-lifting adenoma, submucosal tumors, neuroendocrin tumors, incomplete endoscopic resection (R1) or T1 carcinoma.

Colorectal FTRD is a feasible technique for the treatment of colorectal lesions and represents a minimally invasive alternative for either surgical or conventional endoscopic resection strategies. Clin Endosc 2018;51:103-108

Key Words: Colonoscopy; Colorectal neoplasms; Full-thickness resection device; Over-the-scope-clip

\section{INTRODUCTION}

A novel full-thickness resection device (FTRD; Ovesco, Tuebingen, Germany) based on the over-the-scope-clip (OTSC) system was recently introduced in Europe. To date, the FTRD

Received: June 7, 2017 Revised: September 27, 2017

Accepted: October 20, 2017

Correspondence: Edris Wedi

Department of Gastroenterology and Gastrointestinal Oncology, University Medical Center Göttingen, Robert-Koch-Straße 40, Göttingen 37075, Germany Tel: +49-551-39-66333, Fax: +49-551-39-66333,

E-mail: edris.wedi@med.uni-goettingen.de

(c) This is an Open Access article distributed under the terms of the Creative Commons Attribution Non-Commercial License (http://creativecommons.org/ licenses/by-nc/3.0) which permits unrestricted non-commercial use, distribution, and reproduction in any medium, provided the original work is properly cited. is approved for use in Europe, USA and Australia for the resection of colorectal lesions. The FTRD combines a modified OTSC mounted on the distal tip of the endoscope with an electrocautery snare (Fig. 1A). Endoscopic mucosal resection (EMR) and endoscopic submucosal dissection (ESD) are the current endoscopic treatment options for colorectal adenomas with low-grade dysplasia or high-grade dysplasia, and early mucosal-based cancer. ${ }^{1}$ However, these techniques have limitations in cases of submucosal scarring and fibrosis. In these instances, a full-thickness device could be a valid alternative. Furthermore, patients who would otherwise well qualify for surgical resection but are poor surgical candidates may also be considered for treatment with the FTRD.

The FTRD system consists of a plastic cap $(13 \times 23 \mathrm{~mm})$ 

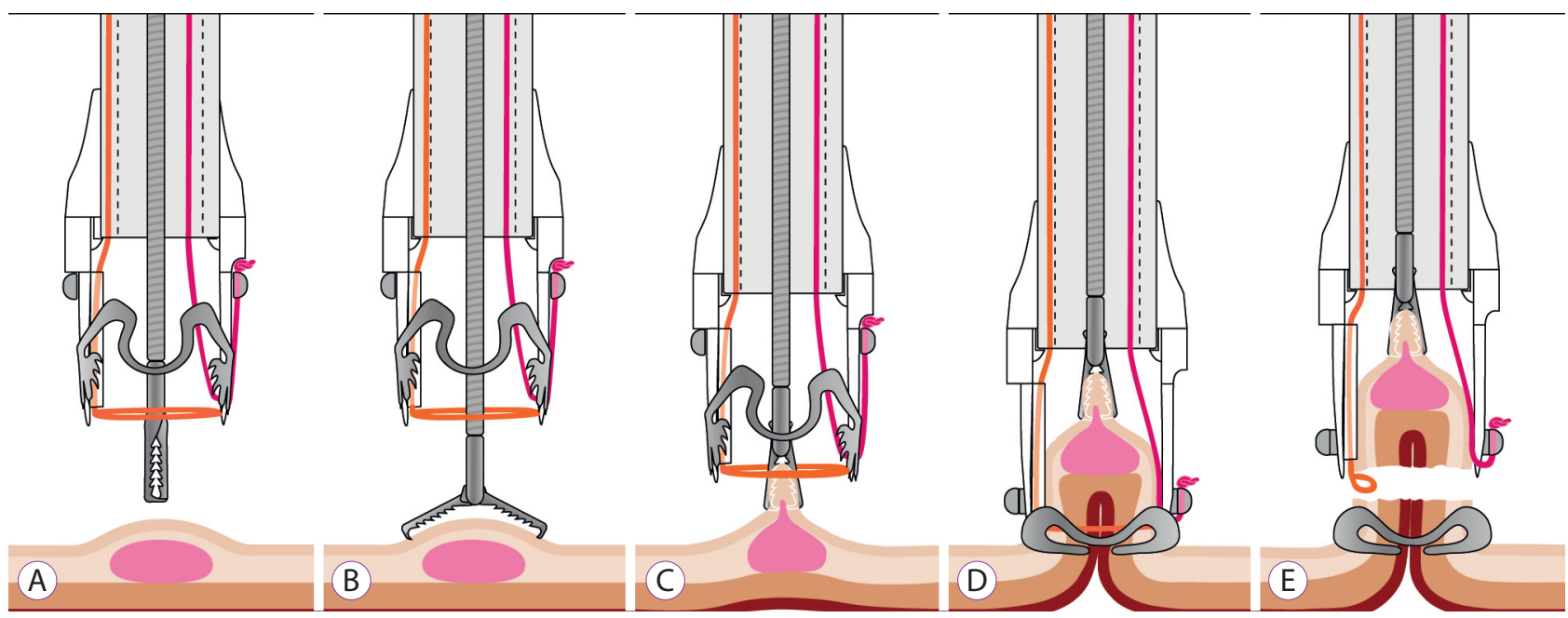

Fig. 1. Step by step description of the full-thickness resection device (FTRD) technique. (A) Components of the FTRD system: the cap, the grasper and the snare. (B) Grasping the lesion with the FTRD grasper. (C) Retrieval of the target tissue into the cap. (D) Release of the over-the-scope-clip. (E) Closure.

that is loaded with an FTRD clip and has a preloaded 14$\mathrm{mm}$ polyfilament polypectomy snare inside the cap (Fig. 1A), as well as ancillary instruments, a tissue grasper, and a high-frequency marking device. In contrast to most endoscopic polypectomy snares, the snare in the FTRD system does not advance through the working channel; instead, the shaft runs on the external side of the scope under a plastic sheath to leave the working channel free for instrumentation. After identifying the target lesion, the circumference of each lesion is premarked with a 1.5-mm Flush knife (Fujifilm, Tokyo, Japan) by using the coagulation setting (VIO 200; ERBE, Tuebingen, Germany, forced coagulation effect 1, 20 W). Next, the colonoscope is withdrawn and the FTRD system is mounted on the scope. Once the scope is re-inserted, the lesion is pulled inside the distal plastic cap by using an appropriate grasper, with the intention of pulling all layers of the colonic wall into the cap (Fig. 1B, C). Then, the FTRD clip is deployed and the electrocautery snare is activated using a monopolar current (VIO 200, Endocut Q 1/5/4), excising the full-thickness tissue captured by the clip (Fig. 1D, E). The specimen is then retrieved, leaving the colonic wall closed by the OTSC. The procedural time in our case series varied from 30 to $90 \mathrm{~min}$. After the procedure, 1 day of fasting and 2 subsequent days of liquid diet were prescribed, and antibiotic prophylaxis with ceftriaxone and metronidazole for 5 days was administrated.

\section{CASE REPORTS}

In this report, technical success was defined as a successful deployment of the OTSC on the target lesion and clinical success was defined as R0 resection in histopathological analysis. Three patients were treated with the FTRD system from December 2014 to September 2015 at the University Hospital of Strasbourg, France. Additionally we performed a comprehensive review of the literature on the use of the FTRD system for the treatment of gastrointestinal (GI) lesions, by using the Medline, Web of Science, and Scopus databases up to December 2016.

\section{Case 1}

The first case involved a 64-year-old male patient with a history of a well-differentiated rectal adenocarcinoma (pT3N1bM0) in 2011, which was treated with neoadjuvant capecitabine-based chemotherapy and radiotherapy, followed by rectosigmoidectomy, with a good response. A colonoscopy performed in November 2014 showed a local recurrence of the adenocarcinoma near the anastomosis. The lesion was located $10 \mathrm{~cm}$ from the anal margin and $5 \mathrm{~cm}$ proximal to the colorectal anastomosis, without any infiltration signs $(15 \times 12$ $\mathrm{mm}$, nongranular type, Paris IIa). Endoscopic ultrasound and magnetic resonance imaging excluded direct infiltration of the muscularis propria or lymph node involvement (Fig. 2A). Computed tomography (CT) scans were negative for metastasis. Following the multidisciplinary tumor board decision, the lesion was resected with the FTRD system (Fig. 2B-E). The $25-\mathrm{mm}$ specimen was confirmed to be a moderately differentiated rectal adenocarcinoma with margins free from malignancy (pT1N0M0, G2, L0, R0) and an infiltration depth of $850 \mu \mathrm{m}$ into the submucosa (sml) in the histopathological analysis. A follow-up at 9 months showed a scar, without local recurrence (Fig. 2F).

\section{Case 2}

The second case is an 89-year old male patient who was 

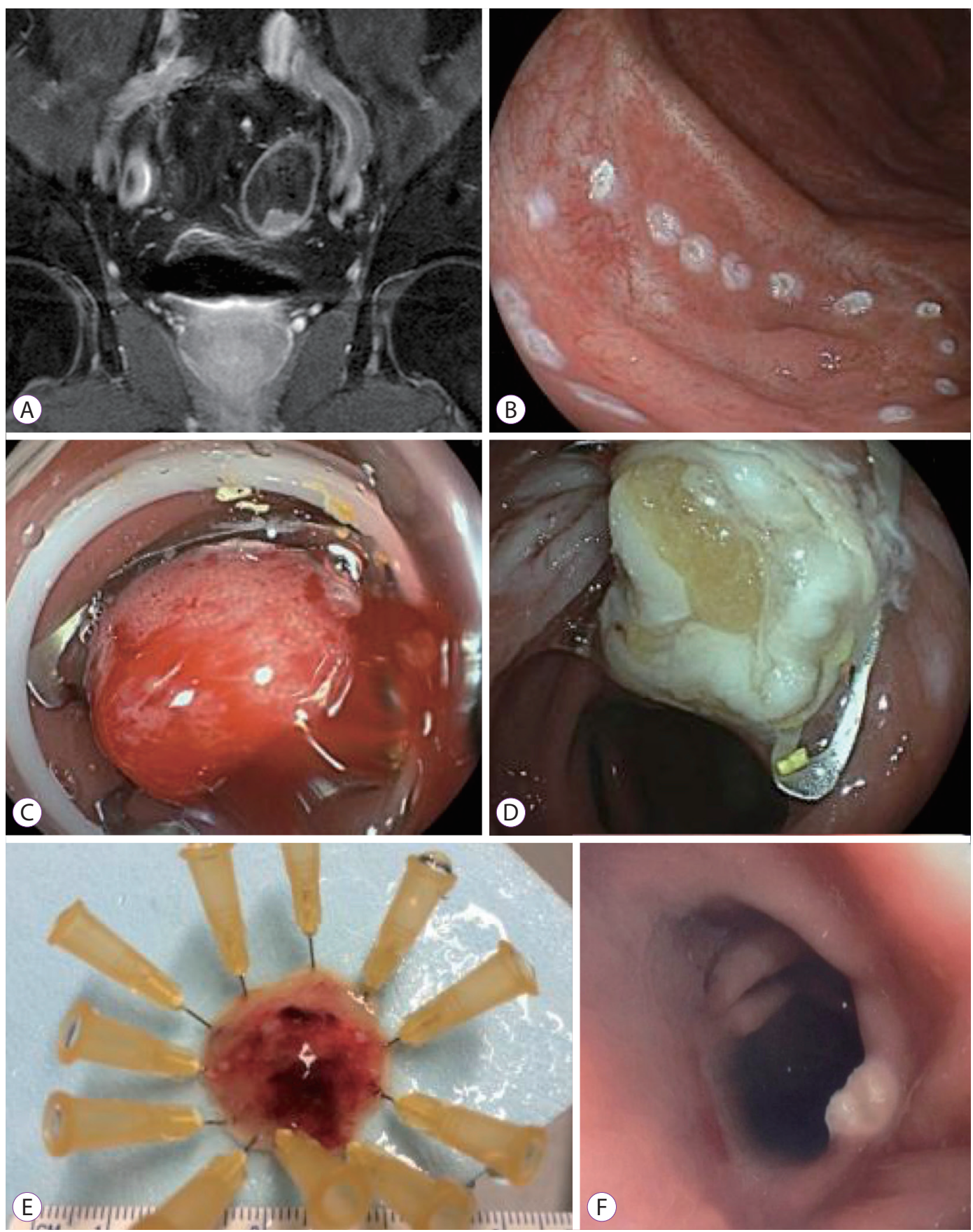

Fig. 2. Case 1: Magnetic resonance imaging (MRI) and endoscopic images. (A) Coronal MRI image, demonstrating local recurrent rectal adenocarcinoma, without muscle layer infiltration. (B) Pre-markage of the lesion with a $1.5 \mathrm{~mm}$ Flush knife (Fujifilm, Tokyo, Japan) using the coagulation setting. (C) Lesion after over-the-scopeclip deployment. (D) Aspect of the mucosa after resection. (E) Resected lesion. (F) Endoscopic view at a follow-up colonoscopy, 15 months status-post endoscopic resection.

referred to our hospital for surveillance colonoscopy of a previously resected pT2N0 rectal cancer in 1998. Colonoscopy showed a $3.0 \times 1.5 \mathrm{~cm}$ laterally spreading lesion, nongranular type (Paris IIa) situated on a colonic fold in the distal transverse colon. ESD wasn't possible due to difficult position and non-lifting sign due to fibrosis. Because of the high risk of perforation of ESD or EMR in this lesion, we converted to the FTRD and completed the resection "en bloc". Histopathological analysis of the $4 \times 2.3 \mathrm{~cm}$ specimen revealed a tubulovillous adenoma with low-grade dysplasia, R0. The further clinical course of the patient was uneventful. 


\section{Case 3}

The third case involved a 39-year-old male patient with a history of Alport syndrome with hypoacusis, ulcerative colitis, deep venous thrombosis, and end-stage renal disease requiring renal transplantation in 1999. This patient was receiving immunosuppressive therapy with tacrolimus and azathioprine. Colonoscopy showed a local recurrence of an adenoma $(1.5 \times 1.8 \mathrm{~cm})$ in the ascending colon with high-grade dysplasia involving an EMR scar, surgical intervention was refused; thus, we planned resection with FTRD. Resection with the FTRD system was performed, and the $3.0 \times 2.3 \mathrm{~cm}$ specimen was confirmed to be a flat tubular adenoma with high-grade dysplasia, R0. Two days after the endoscopic therapy, the patient presented with acute abdominal pain and a CT scan revealed intestinal perforation. Exploratory laparoscopy was carried out, the perforation site was directly adjacent to the site of clip placement, with the FTRD clip still in place, right hemicolectomy was performed. Histopathological analysis of the specimen revealed no adenomatous tissue. The patient recovered completely and was discharged from the hospital on postoperative day 11 .

\section{DISCUSSION}

The presented cases show that endoscopic full-thickness resection of mucosal lesions is an area of increasing interest. The idea of applying a closure tool before performing a resection is innovative, ensures that the GI wall remains sealed, and avoids a potentially challenging perforation closure procedure after resection. ${ }^{2}$ Furthermore, this one-step procedure avoids the possible leakage of contaminated GI content into the abdominal cavity. ${ }^{2}$ Resection with the FTRD seems to have a faster learning curve and is less time consuming compares to ESD or EMR. 3,4

In some case studies, a precursor technique combining standard OTSC application and subsequent snare polypectomy has been described to achieve full-thickness resection. ${ }^{5}$ According to our review, this technique has been used in 20 patients for neuroendocrine tumors (NETs), granular cell tumors of the esophagus, incomplete prior endoscopic resection (R1), adenoma relapse, and submucosal tumors. Full-thickness resection and R0 was obtained in $93.8 \%-100 \%$, and no adverse events were reported..$^{5-7}$ The currently available FTRD system was a logical evolution of this technique combining the OTSC and a monofilament snare in one device.

Seven publications on the FTRD device were found from August 2014 to April 2016, with a total of 52 reported patients (Table 1). Overall, the most frequent indications were lifting or nonlifting adenomas, submucosal tumors, NETs, incomplete endoscopic resection (R1), or T1 carcinomas. ${ }^{8-10}$ Concerning complications, bleeding has been described in $9.1 \%$ of cases. In the series included in our review, the rates of both technical success and clinical success ranged between $75 \%$ and $100 \%$ (Table 1). Richter-Schrag et al. recently published a series of 20 patients with colorectal lesions treated with the FTRD. ${ }^{8}$ The $\mathrm{R} 0$ resection rate was reported to be $80 \%$. They concluded that FTRD is a safe and effective modality for resecting lesions in the lower GI tract; however, they pointed out that the system has some limitations, including in cases of scarring or fibrotic lesions, and in relation to the thickness of the intestinal wall.

One limitation of the FTRD concerns the size of the lesions that can be resected. In animal experiments and in human resection specimens, the maximal reported lesion size was $5.4 \mathrm{~cm}$ (with much smaller lesion sizes reported in normal clinical practice), depending on the thickness, rigidity, and mobility of the GI wall. ${ }^{11}$

The possible complications that can occur with the FTRD are similar to those with the OTSC system, such as false clip application, clip insufficiency, luminal stenosis due to the size of the clip, or fixation of the grasper in between the teeth of the clip. A potentially life-threatening severe complication is grasping and clipping of neighboring intestinal structures, such as large vessels or adjacent organs. These should be taken into consideration when using the device.

In this case series, we presented three cases of resection of complex colorectal lesions with the FTRD system. In two of the three patients, standard resection techniques failed and the FTRD was used as a final endoscopic option before surgery.

To our knowledge, the first patient is the first case in which the FTRD was utilized for the resection of a T1 adenocarcinoma (local recurrence in the region of the previous surgical anastomosis). Lesions situated in the region of a rectal surgical anastomosis, for example, are well known to be difficult to resect surgically because of the complexity of creating a new intestinal anastomosis. Therefore, the FTRD system allows performing a complete resection in a challenging surgical environment while minimizing the procedural morbidity. The second case shows the opportunity to use the FTRD system in patients with advanced age and several comorbidities who are unfit for surgery, and in those with colorectal lesions poorly suited for conventional endoscopic resection techniques.

The complication described in the third case, delayed perforation occurring $>48 \mathrm{~h}$ from the endoscopy procedure, was likely multifactorial in etiology and has not previously been described. The patient had intra-abdominal adhesions due to prior operations and infections (renal transplantation, 


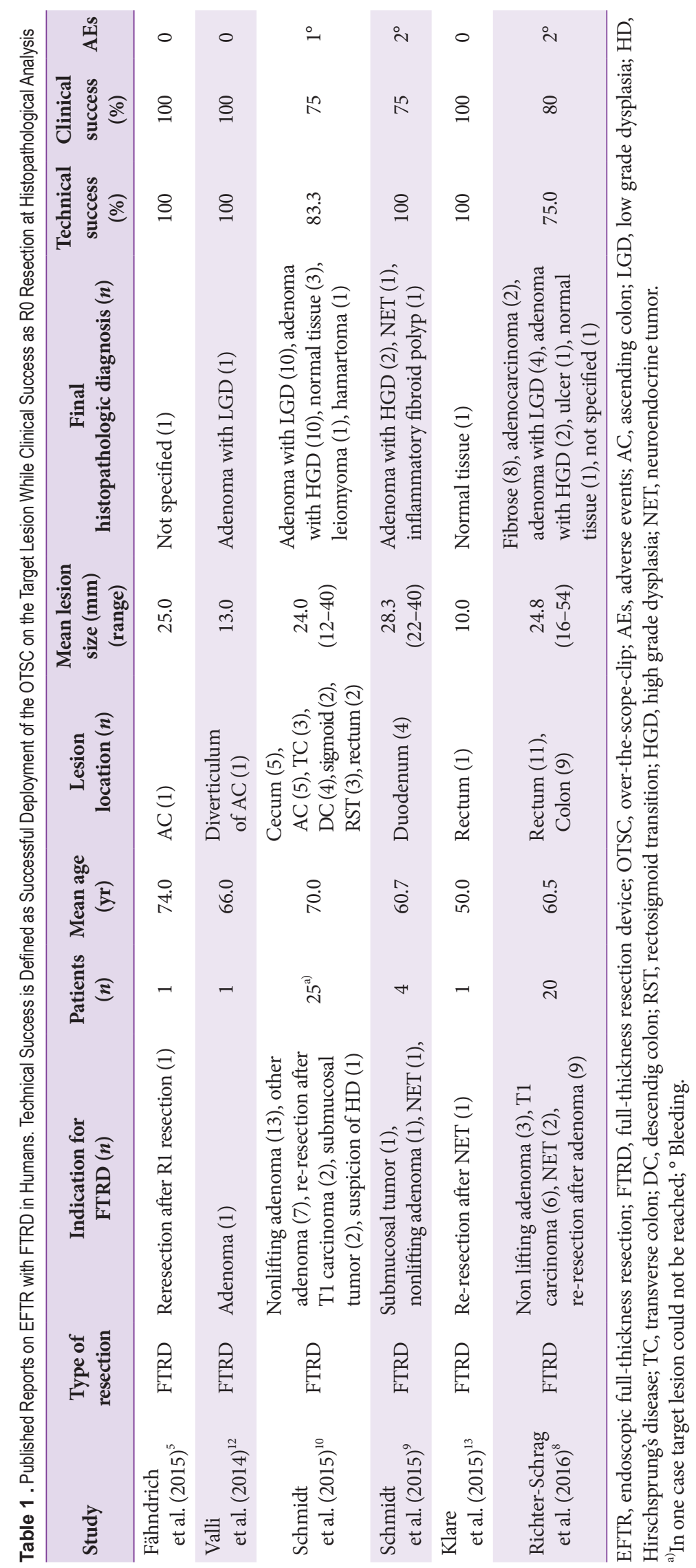


ulcerative rectocolitis, and appendectomy) and was receiving chronic immunosuppressive therapy. The surgical specimen after perforation showed that the perforation site was directly adjacent to the OTSC clip. It is known from the surgical literature that patients under immunosuppression have a sharply increased risk of secondary complications and leakage in the region of the colonic anastomosis. ${ }^{14}$

In conclusion, FTRD pushes the frontiers of endoscopic resection toward transmural, endoluminal, and more complete oncologic resections. However, further research is needed to demonstrate the efficacy and safety of this innovative technique compared with the gold-standard surgical resection.

\section{Conflicts of Interest}

The authors have no financial conflicts of interest.

\section{REFERENCES}

1. Hochberger J, Köhler P, Kruse E, et al. [Endoscopic submucosal dissection]. Internist (Berl) 2013;54:287-301.

2. von Renteln D, Rösch T, Kratt T, Denzer UW, El-Masry M, Schachschal G. Endoscopic full-thickness resection of submucosal gastric tumors Dig Dis Sci 2012;57:1298-1303.

3. Schurr MO, Baur F, Ho CN, Anhoeck G, Kratt T, Gottwald T. Endoluminal full-thickness resection of GI lesions: a new device and technique. Minim Invasive Ther Allied Technol 2011;20:189-192.

4. Hotta K, Oyama T, Shinohara T, et al. Learning curve for endoscop- ic submucosal dissection of large colorectal tumors. Dig Endosc 2010;22:302-306

5. Fähndrich M, Sandmann M. Endoscopic full-thickness resection for gastrointestinal lesions using the over-the-scope clip system: a case series. Endoscopy 2015;47:76-79.

6. Mönkemüller K, Peter S, Toshniwal J, et al. Multipurpose use of the bear claw' (over-the-scope-clip system) to treat endoluminal gastrointestinal disorders. Dig Endosc 2014;26:350-357.

7. Sarker S, Gutierrez JP, Council L, Brazelton JD, Kyanam Kabir Baig KR, Mönkemüller K. Over-the-scope clip-assisted method for resection of full-thickness submucosal lesions of the gastrointestinal tract. Endoscopy $2014 ; 46: 758-761$

8. Richter-Schrag HJ, Walker C, Thimme R, Fischer A. [Full thickness resection device (FTRD). Experience and outcome for benign neoplasms of the rectum and colon]. Chirurg 2016;87:316-325.

9. Schmidt A, Meier B, Cahyadi O, Caca K. Duodenal endoscopic full-thickness resection (with video). Gastrointest Endosc 2015;82:728733.

10. Schmidt A, Bauerfeind P, Gubler C, Damm M, Bauder M, Caca K. Endoscopic full-thickness resection in the colorectum with a novel overthe-scope device: first experience. Endoscopy 2015;47:719-725.

11. Schmidt A, Damm M, Caca K. Endoscopic full-thickness resection using a novel over-the-scope device. Gastroenterology 2014;147:740-742. e2

12. Valli PV, Kaufmann M, Vrugt B, Bauerfeind P. Endoscopic resection of a diverticulum-arisen colonic adenoma using a full-thickness resection device. Gastroenterology 2014;147:969-971.

13. Klare P, Burlefinger R, Neu B, et al. Over-the-scope clip-assisted endoscopic full-thickness resection after incomplete resection of a rectal euroendocrine tumor. Endoscopy 2015;47(Suppl 1) UCTN:E47-E48.

14. Eriksen TF, Lassen CB, Gögenur I. Treatment with corticosteroids and the risk of anastomotic leakage following lower gastrointestinal surgery: a literature survey. Colorectal Dis 2014;16:O154-O160. 